\title{
Convection-enhanced delivery in glioblastoma: a review of preclinical and clinical studies
}

\author{
*Arman Jahangiri, BS, Aaron T. Chin, BS, Patrick M. Flanigan, BS, Rebecca Chen, BS, \\ Krystof Bankiewicz, PhD, and Manish K. Aghi, MD, PhD
}

Department of Neurological Surgery, University of California at San Francisco, San Francisco, California

\begin{abstract}
Glioblastoma is the most common malignant brain tumor, and it carries an extremely poor prognosis. Attempts to develop targeted therapies have been hindered because the blood-brain barrier prevents many drugs from reaching tumors cells. Furthermore, systemic toxicity of drugs often limits their therapeutic potential. A number of alternative methods of delivery have been developed, one of which is convection-enhanced delivery (CED), the focus of this review. The authors describe CED as a therapeutic measure and review preclinical studies and the most prominent clinical trials of CED in the treatment of glioblastoma. The utilization of this technique for the delivery of a variety of agents is covered, and its shortcomings and challenges are discussed in detail.
\end{abstract}

http://thejns.org/doi/abs/10.3171/2016.1.JNS151591

KEY WORDS convection enhanced delivery; glioblastoma; blood-brain barrier; oncology

$\mathrm{G}$ LIOBLASTOMA is the most common malignant brain tumor in adults. Even when aggressively treated with surgery, chemotherapy, radiation, and novel chemotherapies, the median duration of survival remains just shy of 2 years. ${ }^{10}$ While gradually improving over time, the prognosis for glioblastoma remains poor, and novel therapeutic approaches are desperately sought after. ${ }^{18,42,71}$

Part of the lack of efficacy of chemotherapy drugs against glioblastoma results from the inability of most small molecules (98\%) to pass the blood-brain barrier (BBB). ${ }^{50}$ The $\mathrm{BBB}$ usually prevents therapeutic agents with a molecular weight greater than $180 \mathrm{kDa}$ from passing through. To overcome this, intraparenchymal delivery of therapeutic agents has been investigated. Approaches to local drug delivery have included the use of implantable polymers that slowly release drugs ${ }^{37}$ and convection-enhanced delivery (CED) through rigid cannulas and/or soft catheters ${ }^{37}$ Here, we review the latter approach, including its conceptual origins, clinical trial results to date, current challenges, and future directions of the field.

\section{Methods}

A PubMed search was performed, using the phrase "convection-enhanced delivery and glioblastoma," for all years up to 2015. The references of systematic reviews were examined for additional sources. Each article's abstract was reviewed. Our inclusion criteria consisted of preclinical or clinical studies in which CED was studied for the treatment of glioblastoma. These articles were reviewed based on survival outcomes, adverse effects, and methodologies. Studies focusing on physical mechanisms of CED were included, along with articles exploring technological CED advancements. Finally, studies elucidating the physiological properties and barriers in the brain related to drug delivery were used. Eighty-seven articles were chosen for this review.

\section{Results \\ Conceptual Development of CED: 1994-1996}

Researchers seeking to bypass the BBB, which impedes many potentially effective treatments, by pursuing local delivery were initially limited to diffusion-mediated delivery. A compound's diffusion in a given tissue depends mainly on its free concentration gradient and its diffusivity in the tissue. Thus, high-molecular-weight compounds like antibodies and enzymes are unable to diffuse over large distances, and drug distribution is very limited. ${ }^{23}$ For example, it may take up to 3 days for an immunoglobulin

ABBREVIATIONS BBB = blood-brain barrier; $\mathrm{CED}$ = convection-enhanced delivery; $\mathrm{GDL}$ = gadoteridol-loaded liposomes; $\mathrm{HSV}$-TK = herpes simplex virus-thymidine kinase; IL-4 = interleukin-4; OS = overall survival; PFS = progression-free survival; TF = transferrin; TMZ = temozolomide; TRAIL = tumor necrosis factor-related apoptosisinducing ligand.

SUBMITTED July 08, 2015. ACCEPTED January 08, 2016.

INCLUDE WHEN CITING Published online April 1, 2016; DOI: 10.3171/2016.1.JNS151591.

${ }^{*}$ Arman Jahangiri and Aaron T. Chin contributed equally to this work. 
to diffuse $1 \mathrm{~mm}$ from its delivery site, which is far from ideal as a therapeutic method. ${ }^{23}$

CED, initially conceptualized by Bobo et al. in 1994 as a local delivery strategy for the CNS, can overcome these limitations of diffusion-mediated delivery. ${ }^{4} \mathrm{CED}$ involves stereotactically inserting 1 or more rigid cannulas containing soft catheters into tumors through bur holes. The catheters are proximally connected to a syringe pump containing the infusate, while their distal ends protrude out beyond the tip of the cannula(s) into the targeted tumor area, establishing a positive pressure gradient for hours to days.

CED is powered by bulk flow kinetics from pressure gradients, as opposed to the concentration gradients relied upon for standard diffusion-based delivery. Although it was originally thought that infusates move through the interstitial space, there is evidence that molecules might move by other means, such as perivascularly, para-arterially, or through slower axonal transport. ${ }^{27,28,39,47,61,62}$

CED offers several advantages over diffusion-mediated delivery. First, CED improves intratumoral spatial distribution because the pressure gradient allows agents to be infused over a larger volume, more evenly and at higher quantities than in diffusion-based approaches (Fig. 1). ${ }^{52,72,76}$ Second, because CED lacks the steep concentration gradients associated with diffusion-mediated delivery, less toxic doses can be used. ${ }^{32}$ Third, unlike diffusion, CED occurs independent of an agent's molecular weight or diffusivity.

\section{Preclinical Development of CED for Glioblastoma: Summary of Select Studies}

Since the 1994 study of Bobo et al., ${ }^{4}$ CED for gliomas continues to be investigated in preclinical models. Here, we review various noteworthy preclinical studies. Intracerebral CED of topotecan in a rat glioma model demonstrated that 11 of 12 rats treated survived beyond 120 days while the control cohort did not live beyond 26 days. ${ }^{25} \mathrm{An}$ - other study assessed CED of chemotherapies gemcitabine or carboplatin, in which $63 \%$ of animals receiving either agent via CED lived to the 120-day mark, while all control animals receiving phosphate-buffered saline via CED or systemic treatment expired before 26 days. ${ }^{11}$ These studies, while promising, failed to compare CED of a therapy to systemic delivery of a drug with known efficacy. A study in 2004 did that by comparing baseline oral temozolomide (TMZ) efficacy to results achieved with the addition of TRAIL (tumor necrosis factor-related apoptosis-inducing ligand) via CED in athymic mice. This study established that the combination of TRAIL and TMZ prolonged overall survival (OS) compared with TMZ alone.$^{58}$ Moreover, a 2015 study revealed that bevacizumab, a humanized VEGF (vascular endothelial growth factor) antibody, administered via CED, increased survival over intravenous bevacizumab. This result supports further developing CED by showing that current glioblastoma treatments could be more effective via CED. ${ }^{80}$ Other studies published in 2015 assessed the efficacy of CED of agents used to systemically treat glioblastoma such as irinotecan, carboplatin, and cetuximab delivered via nanoparticles and liposomes to allow gradual release after CED., ${ }^{1,26,43}$ These preclinical studies have improved CED techniques and identified agents to infuse via CED, information that has given rise to the multiple clinical trials in patients described below.

\section{Results of CED Clinical Trials 1997-2010}

The results of CED clinical trials published to date are summarized in Table 1. Most trials have used flexible, single-lumen catheters rather than the rigid cannulas with tumor-penetrating catheters proposed in initial preclinical studies. ${ }^{4}$ Of the 14 trials listed, 8 involved conjugated toxins specifically taken up by high-grade glioma cells, 1 involved viruses or liposomes, and 5 involved conventional chemotherapies unable to penetrate the BBB. For trials involving these chemotherapies, optimal cannula positioning safely away from the ventricles is vital to prevent leakage of
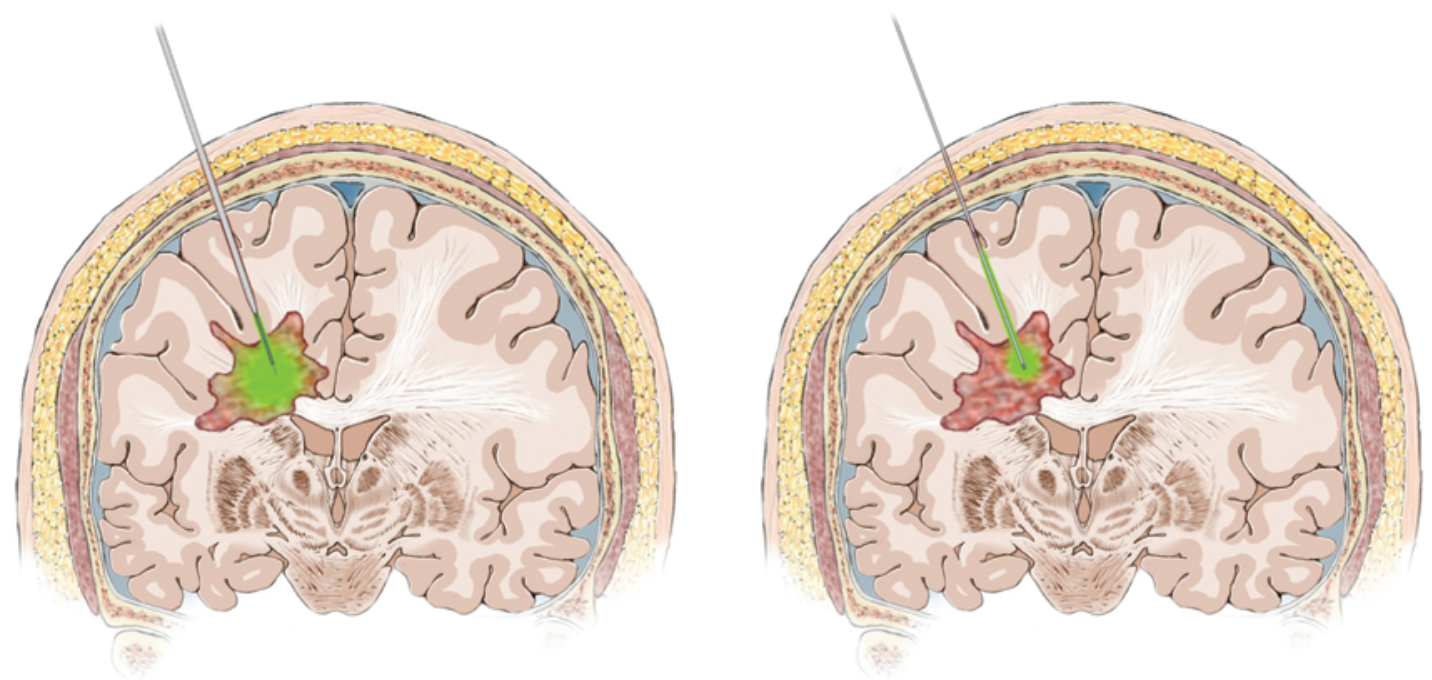

FIG. 1. Artist's illustrations showing the improvement in distribution with CED relative to diffusion occurring with nonconvected injection. Left: Shown is theoretical distribution with minimal associated reflux (green in cannula) with CED. Right: Shown is theoretical reduced distribution with greater reflex (green in cannula) with diffusion occurring with a nonconvected injection. Copyright UCSF Neurosurgery. Published with permission. Figure is available in color online only. 


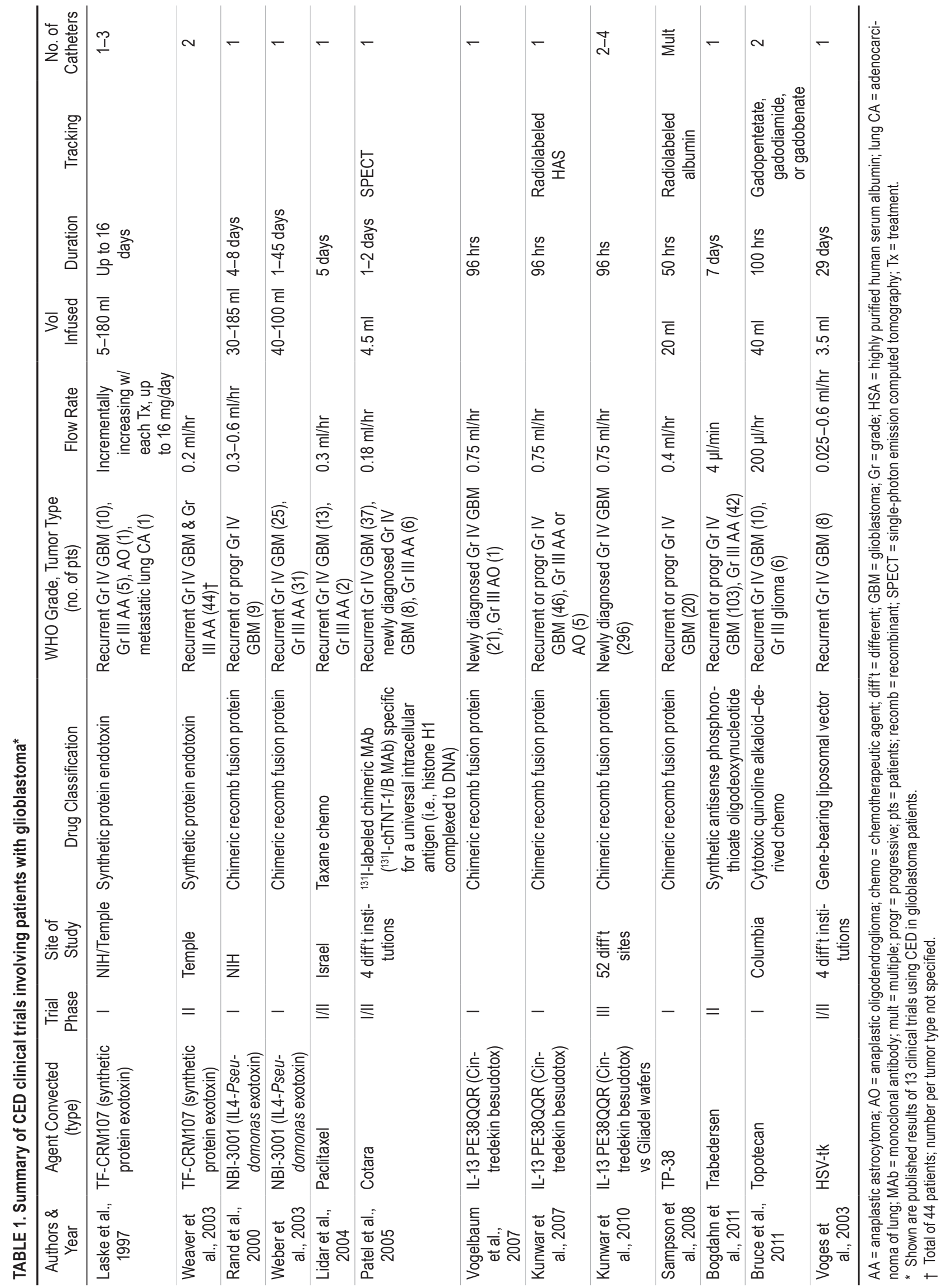


chemotherapeutic agents into the CSF, which could cause aseptic chemical meningitis, ${ }^{7}$ although this complication has never been reported when chemotherapy is delivered into the parenchyma via CED. Below we summarize some important representative examples of these trials.

\section{CED Trials Involving Conjugated Toxins}

With promising results obtained from preclinical models, the first clinical trial was performed in glioblastoma using the targeted toxin TF-CRM107, a human transferrin (TF) conjugated to diphtheria toxin (CRM107) with a point mutation that abrogates its nonspecific binding to mammalian cells. Results (reported in 1997) showed that 9 of 15 patients had tumor size reduction with limited toxicity, the first demonstration of CED feasibility in brain tumor patients. ${ }^{36}$ The Phase II arm for TF-CRM107, reported in 2003, produced slightly less encouraging results with only 31 of 44 patients completing treatment and only 12 of those 31 having a complete or partial radiographic response. Overall, 23 patients had progressive disease, while 21 patients had arrested disease; the median survival was 37 weeks. Toxicity included cerebral edema in $14 \%$ of patients ${ }^{81}$ Unfortunately, the Phase III study involving TF-CRM107, conducted at 40 centers in the US and Europe was aborted because intermediate analysis of 44 patients showed only a $39 \%$ response rate. Timely reporting of aborted trials like the TF-CRM107 Phase III study and thorough analysis of potential sources of failure like cannula placement will be essential for the development of future CED trials.

Interleukin-4 (IL-4) receptor overexpression in malignant glioma cells was taken advantage of through the use of IL-4 Pseudomonas exotoxin (NBI-3001), a chimeric recombinant fusion protein made of IL-4 and Pseudomonas exotoxins. The first of such trials was in 2000 and involved a cohort of 9 patients with glioblastoma, 6 of whom demonstrated tumor necrosis after treatment, a promising response with limited toxicity. ${ }^{55}$ In a similar NBI-3001 trial, a cohort of 6 Grade III astrocytoma patients and 25 glioblastoma patients received CED as their only treatment. The median OS for this study was 8.2 months; patients with glioblastoma had a median OS of 5.8 months. T1-weighted MRI demonstrated a significant amount of tumor necrosis in a majority of these patients. ${ }^{82}$ There was no systemic toxicity, and adverse effects were limited to treatable edema. An additional case study involving a patient with recurrent glioblastoma treated with NBI-3001 in a single infusion showed that the patient survived for 36 months. ${ }^{53}$ The IL-4-Pseudomonas exotoxin fusion protein holds promise as a targeted therapeutic for glioblastoma and is being developed by Medicenna Therapeutics.

Epidermal growth factor receptor (EGFR) overexpression is another feature of glioblastoma that can be therapeutically targeted. TP-38 is a chimeric protein containing a Pseudomonas exotoxin and a TGF- $\alpha$ binding domain that binds tightly to EGFR. Its binding delivers the exotoxin and induces apoptosis. In a study of 20 patients with recurrent or progressive malignant brain tumors (17 glioblastomas), the median time to progression was 14.9 weeks, and the median OS was 28 weeks. However, many patients experienced significant leaks into the ventricles or subarachnoid space resulting in failed intraparenchy- mal distribution. Although the treatment was considered safe, this trial revealed the importance of real-time CED monitoring. ${ }^{63,66}$ In a case study involving a patient with multiply recurrent glioblastoma, TP-38 prevented tumor progression for over 43 months, with PET revealing hypometabolic tumor activity, suggesting treatment response. ${ }^{66}$

Beyond the Phase III TF-CRM107 study mentioned above, the only other randomized Phase III CED clinical trial to date studied IL13-PE38QQR (Cintredekin besudotox), a chimeric protein made up of IL-13 and a truncated form of Pseudomonas exotoxin A (PE38QQR), which is taken up by glioma cells expressing the IL-13 receptor alpha 2 chain (IL-13R $\alpha 2$ ). This trial (named the PRECISE Trial) enrolled 296 patients; one arm received IL13PE38QQR via CED through catheters implanted after craniotomy for resection 96 hours earlier, and the other received CCNU-containing Gliadel wafers implanted in the walls of the resection cavity after craniotomy. Although no OS benefit was found for CED of IL13-PE38QQR relative to Gliadel wafers, ${ }^{34}$ the study was powered to detect greater than 50\% survival benefit over the Gliadel-treated control arm, and detecting a smaller but meaningful difference would have required a larger cohort, significantly elevating the trial's cost. A follow-up study noted that only $68 \%$ of catheter placements were performed per protocol, suggesting that variability in catheter positioning may have adversely impacted results. ${ }^{46}$ The authors also commented that excluding patients with lower IL-13 receptor levels might have improved efficacy. Despite failure to improve OS, the prespecified objective for efficacy, improved progression-free survival (PFS) (18 vs 11 weeks, $p$ $=0.0008)$ was reported ${ }^{46}$

\section{CED Trials Involving Conventional Chemotherapy}

Paclitaxel, a chemotherapeutic agent unable to cross the BBB, was studied in a CED trial for the treatment of highgrade gliomas published in 2004 by Lidar et al. ${ }^{38}$ Thirteen glioblastoma and 2 anaplastic astrocytoma patients with recurrent disease received a total of 20 cycles of paclitaxel via CED. Eleven of 15 patients showed an imaging response, and the median survival was 7.5 months..$^{38}$

Camptothecin class drugs inhibit topoisomerase I, thereby disrupting DNA replication and triggering apoptosis. Topotecan, a member of this class, was used in a CED trial with 16 patients (10 glioblastoma, 6 anaplastic astrocytoma). ${ }^{6}$ The trial's median OS and PFS were 60 and 23 weeks, respectively. ${ }^{6}$ Topotecan was not detected in patient serum, and patients receiving the highest dose suffered only minor neurological deficits.

\section{CED Trials Involving Liposomes or Viruses}

Implanted liposomes, spherically shaped artificial vesicles bearing a lamellar-phase lipid bilayer, can be used for gradual therapeutic delivery. Nanoparticles within the liposomes can be modified to control drug release and improve target-site specificity. ${ }^{73,79}$ Liposomes can also undergo endocytosis or phagocytosis, allowing intracellular delivery of drugs that cannot pass across the cell membrane..$^{14,21,85}$

Liposomes have been used to deliver genes to tumor cells in several trials. Initial attempts at gene delivery to 
tumor cells involved implanting vector-producing cells generating retroviruses carrying the herpes simplex virusthymidine kinase $(H S V-T K)$ gene. ${ }^{29,54}$ After these attempts failed to produce sufficient tumor cell transduction, intratumoral delivery of nonreplicating adenoviruses containing HSV-TK without convection was studied in clinical trials. ${ }^{12,13}$ The lack of distribution sufficiently beyond the injection site in these studies created an interest in $H S V$ $T K$ gene delivery via CED. In 2003, Voges et al. reported results from a trial in which the $H S V$-TK gene in liposomes was delivered to glioblastomas via CED. ${ }^{22,78}$ This study involved 8 patients, who had a median survival of 28.1 weeks, with no morbidity witnessed from the procedure. Two of 8 patients had tumor reduction over $50 \%$, although the distribution volume was small in this trial. Another study used liposomes carrying nonreplicating Semliki Forest virus with IL-12, an agent that activates natural killer cells, although the results were inconclusive. ${ }^{56}$ Further evaluation of liposomal delivery of therapeutics via CED is clearly warranted.

\section{Challenges Associated With CED}

Although CED holds considerable promise in neurological drug delivery, clinical trials have had limited success. Reasons identified for these failures are summarized below.

\section{Choice of Agent for CED}

While considerable attention has been given to technological development of CED (e.g., cannula design and infusion rates), the choice of agent to be delivered is exceedingly important in dictating the success of CED. In particular, the inclination to use particularly toxic agents for CED based on the presumption that local delivery ensures the agent only gets to the tumor must be avoided. This is because of the need to target not just the tumor but also the infiltrating tumor cells within the peritumoral white matter, which also contains normal brain cells. Thus the delivered agent must possess a wide therapeutic index. Many CED trials have therefore employed molecularly targeted agents whose toxicity is limited to tumor cells. As is the case for systemically administered agents, Phase 0 clinical trials may be needed to narrow the large number of agents being considered for CED. In Phase 0 trials, subtherapeutic doses of an agent are given to a small number of patients, followed by tissue procurement to confirm that the agent's target has been inhibited..$^{33}$ For CED, this would involve a craniotomy after CED to confirm achievement of 2 CED goals: target inhibition by the drug and sufficient intratumoral distribution of the drug by CED.

\section{Cannula Design}

Several improvements in cannula design made over the original rigid cannulas together allow for increased infusion rate, reduced reflux, and increased infusate distribution. Rounded-tip cannulas, for example, were introduced to decrease tissue pressure and thereby reduce tissue damage-related reflux. ${ }^{83}$ The step cannula (Fig. 2) has a distal tip that is smaller in diameter than the rest of the cannula; this design offers the maneuverability of large-di- ameter cannulas with the higher infusion rates afforded by small-diameter cannulas..$^{31,84}$ A single patient study using a recessed-step cannula (step cannula with outer reinforcement) and robot-guided infusions reported $95 \%$ tumor volume coverage. ${ }^{2}$

Multiple modifications have been attempted to improve flow from the cannula. Cannulas were designed with multiple catheters at their tips, which would have lowered infusion rates at each opening. Unfortunately they were ineffective, since most of the infusate was distributed via the proximal tip. ${ }^{52}$ Hollow-tipped cannulas with multiple nano-sized openings for infusate delivery showed in a murine model 3 times higher distribution of infusate compared with single opening cannulas. ${ }^{48}$ Additionally, the balloon cannula (Fig. 2) allows for a large amount of surface area contact within the tumor, confirmed by a canine model showing $90 \%$ infusate distribution with its use. ${ }^{49}$

Longer-term CED will also benefit from cannulas with flexible catheters (Fig. 2), which have been used in most clinical trials to date (Table 1). These cannulas are rigid during insertion due to their rigid interior stylet but become flexible after they have been inserted and the stylet has been removed. These cannulas could allow long infusions in mobile patients, and implantation for up to 5 days has been approved. Cannulas composed of biocompatible materials such as carbothane have been shown to be suitable for even longer-term insertion, with homogenous distribution of infusates and adequate delivery rates $(5 \mu \mathrm{l} /$ min) without reflux or tissue adherence. , $^{3,41,70}$

\section{Cannula Placement}

Retrospective modeled distribution analysis of the PRECISE trial suggested that less than $50 \%$ of the patients had optimal cannula positioning, which could explain the lack of survival benefit in the trial, given that there was a positive correlation between longer survival and cannula placement considered ideal on the basis of modeling. ${ }^{34} \mathrm{Al}-$ though there are numerous assumptions in this analysis, further study and modeling of optimal cannula positioning is clearly warranted. Concepts related to optimal cannula positioning that are fully validated should be incorporated into future CED clinical trials, including concepts lacking in the trials summarized above, such as software algorithms for cannula positioning, mandating formal training in cannula positioning for neurosurgeons participating in these trials, and identifying whether there is a neurosurgical learning curve for cannula positioning that should be accounted for by mandating that neurosurgeons participating in Phase III CED trials have prior experience with Phase I and II CED trials to ensure that Phase III trials are adequately able to assess efficacy. ${ }^{68}$ These stringent criteria may initially limit the generalizability of CED and restrict it to specific providers or specific centers, but demonstrated efficacy of CED in a trial designed with these considerations in mind would likely over time increase the commitment of other centers and providers to obtain the platforms and technical skill needed to implement CED.

\section{Intratumoral Penetration}

The heterogeneous nature of tumors makes homogeneous delivery of therapeutic agents challenging. Several 


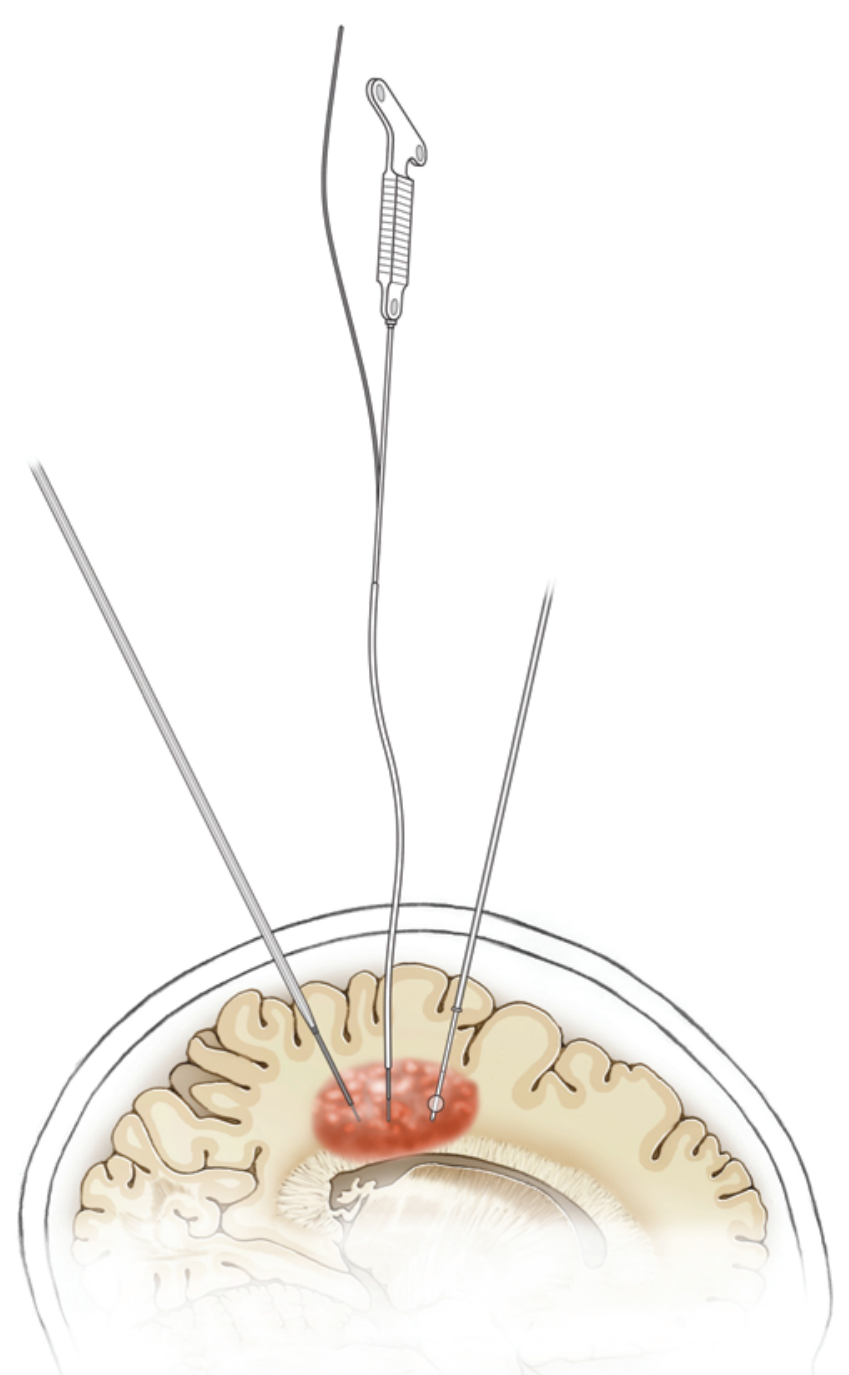

FIG. 2. Artist's illustration of cannula types for CED. Shown are 3 representative CED cannulas infiltrating a tumor to provide CED. From posterior to anterior, these cannulas include: 1) step-down cannula whose tip narrows all the way down to its catheter to reduce reflux, 2) a cannula with a flexible catheter, which is rigid during insertion due to the presence on the inside of a rigid stylet but becomes flexible after the cannula has been inserted and the rigid stylet is removed to allow long infusions in mobile patients; and 3) balloon-tip cannula with a balloon inflating upstream of the infusion occurring at the tip to reduce reflux. Copyright UCSF Neurosurgery. Published with permission. Figure is available in color online only.

physiological and physical barriers prevent full tumor distribution. One physiological barrier is that certain tumor zones metabolize agents faster than others, while a physical barrier can be the aberrant blood vessel growth and increased intercapillary spaces that create differential rates of clearance of agents. ${ }^{17,24,69}$ Modification of therapeutic agents can improve tumoral distribution. For example, cationic infusates favorably "stick" to tumor tissue..$^{59}$ Small agents and nanoparticles may efficiently penetrate tumors by CED because the infusion creates transient edema that increases the extracellular space through which these therapeutics move. ${ }^{6174}$ Finally, computational modeling may improve intratumoral penetration by CED by allowing accurate predictions of concentration distribution. ${ }^{20}$

\section{Reflux}

A major concern with CED is infusate reflux, which has been noted in several trials. ${ }^{45}$ Reflux occurs when the pressure gradient between the cannula and the tumor region equalizes, resulting in the loss of drug flow into the target mass. Three variables have been suggested to contribute to reflux: tissue disruption at the tip of the cannula, infusion rate, and cannula diameter.

Tissue disruption causes reflux when a cannula is inserted too deeply into tissue, if there is a brain shift after the cannula has already been inserted in the brain, or if biopsies are taken at the cannula tip prior to infusing. Tissue disruption potentiates formation and subsequent filling of a cavity, creating a pressure reservoir that ultimately causes backflow up the catheter and outside its wall. Softer cannulas and catheters (Fig. 2) and quick catheter placement reduce brain shift-induced tissue disruption. ${ }^{4,19,52}$

High infusion rates increase reflux risk because pressure caused by the infusion process may exceed interstitial pressure sufficiently to promote reflux. ${ }^{44}$ Fortunately, modern cannula design described below has allowed infusion rates up to $50 \mu \mathrm{l} / \mathrm{min}$ without reflux. ${ }^{8,83}$ However, infusion rates of $3 \mu \mathrm{l} / \mathrm{min}$ or higher can drive infusate into the subarachnoid and ventricular space, causing adverse effects $^{15}$ - underscoring the importance of safe target selection. In designing CED protocols, infusion rate and cannula diameter need to be considered together as they interact such that each cannula diameter and flow rate will be associated with a specific predicted backflow distance along the cannula. ${ }^{45}$

\section{Tracking Infusate Delivery}

None of the trials described above assessed the efficacy of delivery. Two preclinical studies, ${ }^{16,30}$ case reports of 1 or 2 patients, ${ }^{40,60,64}$ and more recent clinical trials whose results have not yet been published have tracked infusate delivery by mixing MRI contrast agents like gadoliniumDPTA and gadoteridol-loaded liposomes (GDL) with infusate and using real-time intraoperative imaging in a surgical MRI suite (NCT02022644 and NCT01156584, ClinicalTrials.gov). This approach has 3 benefits: 1) validation of accurate cannula placement; 2) tracking of agent distribution within the tumor to allow identification of leakage as was done with GDL in an animal study $;{ }^{75}$ and 3) real-time adjustments, including choice of extra targets.

Tracking delivery by mixing the therapeutic agent with a contrast agent assumes that the two agents spread through the tumor similarly. Linking drug to a molecule that can be imaged, assuming the linkage does not disrupt drug efficacy, would address this issue. When patients cannot tolerate gadolinium, T2-weighted imaging can track infusate delivery, although glioblastomas often start with T2 hyperintense regions that would preclude such an approach. ${ }^{57,65,67}$ Validation of these methods for tracking infusate delivery may eventually lead to mandating these measures in Phase III trials.

\section{Cost of Procedure}

For the moment, the cost of CED, which includes time 
in the operating room and/or MRI, cannulas and the platforms needed to place them, infusates, time of the staff performing the procedure, and associated inpatient stays, may limit its applicability to smaller Phase I trials and Phase III trials like PRECISE, for which the corporate sponsor had sufficient funds to conduct an appropriately powered study. But, as with any medical innovation, procedural costs will decline as more companies enter the field and as innovation increases cost-efficiency. For example, the ClearPoint navigation platform (MRI Interventions), which is commonly used in CED, began with a skull mount frame requiring skull exposure comparable to craniotomy, but recently a scalp mount frame has been developed, allowing the cannula to be passed through a nick in the skin, saving an hour of procedural time and reducing infection risk and cost.

Attaching CED to other procedures can allow for insurance coverage of operating room time and hospital stay, but only if that procedure is performed at the same time as CED, which is challenging, because a biopsy, while helpful for confirming tumor recurrence at the site of CED, can create blood products that adversely impact CED, and a craniotomy immediately before CED, although used in the PRECISE trial, creates a cavity, which allows reflux.

\section{Postprocedural Imaging}

The optimal endpoint for CED remains unclear. Until we develop ports that can be implanted long-term through which patients can receive multiple rounds of CED over a prolonged period of time, CED will be a one-time treatment, with treatment durability dependent on the agent being convected-lower for conventional chemotherapy but higher for chemotherapeutic agents in liposomes or replicating viruses. Thus, short-term imaging response such as reduced enhancement or FLAIR may be needed as a response metric alongside conventional parameters, such as PFS or OS, for these trials. The fact that IL13-PE38QQR affected PFS but not $\mathrm{OS}^{34}$ in the PRECISE trial underscores the fact that short-term metrics may better reflect CED success until technology renders multiple CED treatments per patient more feasible. Allowing multiple treatments may enable CED to impact conventional metrics like OS, because unlike agents like bevacizumab, which similarly impact PFS but not OS, ${ }^{9}$ the impact of CED of an agent like IL13-PE38QQR on PFS rather than OS may reflect reduced intratumoral persistence of the agent rather than the tumor evolving resistance to the therapy.

\section{Future Directions}

Here, we have outlined numerous technical challenges that need to be met to overcome the issues encountered with the use of CED to treat glioblastoma to date. Another consideration that will be important to prioritize going forward is that durable CED efficacy might require long-term convection at set intervals for months, as is often required for systemically administered chemotherapy to be effective for nonbrain tumors. The success of such a strategy may require implantable ports that can be cannulated to receive CED in an outpatient setting. Finally, before we can assess the efficacy of an agent delivered via CED, technical reproducibility must be achieved.

\section{Conclusions}

CED bypasses the BBB and reduces systemic toxicity. It has several advantages compared with diffusion-mediated delivery, as pressure gradients allow agents to be disturbed over larger volumes and more evenly at higher quantities, and its distribution is not limited by the drug's physical characteristics. Thus, antitumor drugs that are often toxic systemically can be delivered at higher concentrations with excellent intratumoral distribution. Several therapies have been shown to be safe and somewhat effective in preclinical and clinical studies. Discouraging results from the two randomized Phase III studies conducted to date revealed technical shortcomings that need to be addressed to allow CED to fulfill its therapeutic potential. Overall, CED holds promise for treating glioblastoma and warrants further preclinical and clinical development.

\section{References}

1. Arshad A, Yang B, Bienemann AS, Barua NU, Wyatt MJ, Woolley M, et al: Convection-enhanced delivery of carboplatin PLGA nanoparticles for the treatment of glioblastoma. PLoS One 10:e0132266, 2015

2. Barua NU, Lowis SP, Woolley M, O'Sullivan S, Harrison R, Gill SS: Robot-guided convection-enhanced delivery of carboplatin for advanced brainstem glioma. Acta Neurochir (Wien) 155:1459-1465, 2013

3. Bienemann A, White E, Woolley M, Castrique E, Johnson DE, Wyatt M, et al: The development of an implantable catheter system for chronic or intermittent convection-enhanced delivery. J Neurosci Methods 203:284-291, 2012

4. Bobo RH, Laske DW, Akbasak A, Morrison PF, Dedrick RL, Oldfield EH: Convection-enhanced delivery of macromolecules in the brain. Proc Natl Acad Sci U S A 91:2076-2080, 1994

5. Bogdahn U, Hau P, Stockhammer G, Venkataramana NK, Mahapatra AK, Suri A, et al: Targeted therapy for high-grade glioma with the TGF- $\beta 2$ inhibitor trabedersen: results of a randomized and controlled phase IIb study. Neuro Oncol 13:132-142, 2011

6. Bruce JN, Fine RL, Canoll P, Yun J, Kennedy BC, Rosenfeld $\mathrm{SS}$, et al: Regression of recurrent malignant gliomas with convection-enhanced delivery of topotecan. Neurosurgery 69:1272-1280, 2011

7. Chamberlain MC, Kormanik PA, Barba D: Complications associated with intraventricular chemotherapy in patients with leptomeningeal metastases. J Neurosurg 87:694-699, 1997

8. Chen MY, Lonser RR, Morrison PF, Governale LS, Oldfield EH: Variables affecting convection-enhanced delivery to the striatum: a systematic examination of rate of infusion, cannula size, infusate concentration, and tissue-cannula sealing time. J Neurosurg 90:315-320, 1999

9. Chinot OL, Wick W, Mason W, Henriksson R, Saran F, Nishikawa R, et al: Bevacizumab plus radiotherapy-temozolomide for newly diagnosed glioblastoma. N Engl J Med 370:709-722, 2014

10. Clark AJ, Lamborn KR, Butowski NA, Chang SM, Prados $\mathrm{MD}$, Clarke JL, et al: Neurosurgical management and prognosis of patients with glioblastoma that progresses during bevacizumab treatment. Neurosurgery 70:361-370, 2012

11. Degen JW, Walbridge S, Vortmeyer AO, Oldfield EH, Lonser RR: Safety and efficacy of convection-enhanced delivery of gemcitabine or carboplatin in a malignant glioma model in rats. J Neurosurg 99:893-898, 2003

12. Dewey RA, Morrissey G, Cowsill CM, Stone D, Bolognani F, Dodd NJ, et al: Chronic brain inflammation and persistent 
herpes simplex virus 1 thymidine kinase expression in survivors of syngeneic glioma treated by adenovirus-mediated gene therapy: implications for clinical trials. Nat Med 5:1256-1263, 1999

13. Eck SL, Alavi JB, Alavi A, Davis A, Hackney D, Judy K, et al: Treatment of advanced CNS malignancies with the recombinant adenovirus H5.010RSVTK: a phase I trial. Hum Gene Ther 7:1465-1482, 1996

14. Felgner JH, Kumar R, Sridhar CN, Wheeler CJ, Tsai YJ, Border R, et al: Enhanced gene delivery and mechanism studies with a novel series of cationic lipid formulations. J Biol Chem 269:2550-2561, 1994

15. Fiandaca MS, Forsayeth JR, Dickinson PJ, Bankiewicz KS: Image-guided convection-enhanced delivery platform in the treatment of neurological diseases. Neurotherapeutics 5:123-127, 2008

16. Gimenez F, Krauze MT, Valles F, Hadaczek P, Bringas J, Sharma N, et al: Image-guided convection-enhanced delivery of GDNF protein into monkey putamen. Neuroimage 54 (Suppl 1):S189-S195, 2011

17. Groothuis DR, Ward S, Itskovich AC, Dobrescu C, Allen CV, Dills C, et al: Comparison of ${ }^{14} \mathrm{C}$-sucrose delivery to the brain by intravenous, intraventricular, and convectionenhanced intracerebral infusion. J Neurosurg 90:321-331, 1999

18. Grossman SA, O’Neill A, Grunnet M, Mehta M, Pearlman JL, Wagner H, et al: Phase III study comparing three cycles of infusional carmustine and cisplatin followed by radiation therapy with radiation therapy and concurrent carmustine in patients with newly diagnosed supratentorial glioblastoma multiforme: Eastern Cooperative Oncology Group Trial 2394. J Clin Oncol 21:1485-1491, 2003

19. Guarnieri M, Carson BS, Khan A, Penno M, Jallo GI: Flexible versus rigid catheters for chronic administration of exogenous agents into central nervous system tissues. J Neurosci Methods 144:147-152, 2005

20. Haar PJ, Chen ZJ, Fatouros PP, Gillies GT, Corwin FD, Broaddus WC: Modelling convection-enhanced delivery in normal and oedematous brain. J Med Eng Technol 38:76-84, 2014

21. Hillaireau H, Couvreur P: Nanocarriers' entry into the cell: relevance to drug delivery. Cell Mol Life Sci 66:2873-2896, 2009

22. Jacobs A, Voges J, Reszka R, Lercher M, Gossmann A, Kracht L, et al: Positron-emission tomography of vector-mediated gene expression in gene therapy for gliomas. Lancet 358:727-729, 2001

23. Jain RK: Delivery of novel therapeutic agents in tumors: physiological barriers and strategies. J Natl Cancer Inst 81:570-576, 1989

24. Jain RK: Physiological barriers to delivery of monoclonal antibodies and other macromolecules in tumors. Cancer Res 50 (3 Suppl):814s-819s, 1990

25. Kaiser MG, Parsa AT, Fine RL, Hall JS, Chakrabarti I, Bruce JN: Tissue distribution and antitumor activity of topotecan delivered by intracerebral clysis in a rat glioma model. Neurosurgery 47:1391-1399, 2000

26. Kaluzova M, Bouras A, Machaidze R, Hadjipanayis CG: Targeted therapy of glioblastoma stem-like cells and tumor nonstem cells using cetuximab-conjugated iron-oxide nanoparticles. Oncotarget 6:8788-8806, 2015

27. Kells AP, Forsayeth J, Bankiewicz KS: Glial-derived neurotrophic factor gene transfer for Parkinson's disease: anterograde distribution of AAV2 vectors in the primate brain. Neurobiol Dis 48:228-235, 2012

28. Kells AP, Hadaczek P, Yin D, Bringas J, Varenika V, Forsayeth J, et al: Efficient gene therapy-based method for the delivery of therapeutics to primate cortex. Proc Natl Acad Sci U S A 106:2407-2411, 2009
29. Klatzmann D, Valéry CA, Bensimon G, Marro B, Boyer O, Mokhtari K, et al: A phase I/II study of herpes simplex virus type 1 thymidine kinase "suicide" gene therapy for recurrent glioblastoma. Hum Gene Ther 9:2595-2604, 1998

30. Krauze MT, Forsayeth J, Park JW, Bankiewicz KS: Real-time imaging and quantification of brain delivery of liposomes. Pharm Res 23:2493-2504, 2006

31. Krauze MT, Saito R, Noble C, Tamas M, Bringas J, Park JW, et al: Reflux-free cannula for convection-enhanced highspeed delivery of therapeutic agents. J Neurosurg 103:923929, 2005

32. Kroll RA, Pagel MA, Muldoon LL, Roman-Goldstein S, Neuwelt EA: Increasing volume of distribution to the brain with interstitial infusion: dose, rather than convection, might be the most important factor. Neurosurgery 38:746-754, 1996

33. Kummar S, Rubinstein L, Kinders R, Parchment RE, Gutierrez ME, Murgo AJ, et al: Phase 0 clinical trials: conceptions and misconceptions. Cancer J 14:133-137, 2008

34. Kunwar S, Chang S, Westphal M, Vogelbaum M, Sampson $\mathrm{J}$, Barnett G, et al: Phase III randomized trial of CED of IL13-PE38QQR vs Gliadel wafers for recurrent glioblastoma. Neuro Oncol 12:871-881, 2010

35. Kunwar S, Prados MD, Chang SM, Berger MS, Lang FF, Piepmeier JM, et al: Direct intracerebral delivery of cintredekin besudotox (IL13-PE38QQR) in recurrent malignant glioma: a report by the Cintredekin Besudotox Intraparenchymal Study Group. J Clin Oncol 25:837-844, 2007

36. Laske DW, Youle RJ, Oldfield EH: Tumor regression with regional distribution of the targeted toxin TF-CRM107 in patients with malignant brain tumors. Nat Med 3:1362-1368, 1997

37. Lesniak MS, Brem H: Targeted therapy for brain tumours Nat Rev Drug Discov 3:499-508, 2004

38. Lidar Z, Mardor Y, Jonas T, Pfeffer R, Faibel M, Nass D, et al: Convection-enhanced delivery of paclitaxel for the treatment of recurrent malignant glioma: a phase I/II clinical study. J Neurosurg 100:472-479, 2004

39. Lieberman DM, Laske DW, Morrison PF, Bankiewicz KS, Oldfield EH: Convection-enhanced distribution of large molecules in gray matter during interstitial drug infusion. J Neurosurg 82:1021-1029, 1995

40. Lonser RR, Warren KE, Butman JA, Quezado Z, Robison RA, Walbridge $S$, et al: Real-time image-guided direct convective perfusion of intrinsic brainstem lesions. Technical note. J Neurosurg 107:190-197, 2007

41. Lopez KA, Tannenbaum AM, Assanah MC, Linskey K, Yun J, Kangarlu A, et al: Convection-enhanced delivery of topotecan into a PDGF-driven model of glioblastoma prolongs survival and ablates both tumor-initiating cells and recruited glial progenitors. Cancer Res 71:3963-3971, 2011

42. Louis DN, Ohgaki H, Wiestler OD, Cavenee WK, Burger PC, Jouvet A, et al: The 2007 WHO classification of tumours of the central nervous system. Acta Neuropathol 114:97-109, 2007

43. Mendiburu-Eliçabe M, Gil-Ranedo J: Combination therapy of intraperitoneal rapamycin and convection- enhanced delivery of nanoliposomal CPT-11 in rodent orthotopic brain tumor xenografts. Curr Cancer Drug Targets 15:352-362, 2015

44. Morrison PF, Chen MY, Chadwick RS, Lonser RR, Oldfield $\mathrm{EH}$ : Focal delivery during direct infusion to brain: role of flow rate, catheter diameter, and tissue mechanics. Am J Physiol 277:R1218-R1229, 1999

45. Morrison PF, Lonser RR, Oldfield EH: Convective delivery of glial cell line-derived neurotrophic factor in the human putamen. J Neurosurg 107:74-83, 2007

46. Mueller S, Polley MY, Lee B, Kunwar S, Pedain C, Wem- 
bacher-Schröder E, et al: Effect of imaging and catheter characteristics on clinical outcome for patients in the PRECISE study. J Neurooncol 101:267-277, 2011

47. Nedergaard M: Neuroscience. Garbage truck of the brain. Science 340:1529-1530, 2013

48. Oh S, Odland R, Wilson SR, Kroeger KM, Liu C, Lowenstein PR, et al: Improved distribution of small molecules and viral vectors in the murine brain using a hollow fiber catheter. $\mathbf{J}$ Neurosurg 107:568-577, 2007

49. Olson JJ, Zhang Z, Dillehay D, Stubbs J: Assessment of a balloon-tipped catheter modified for intracerebral convection-enhanced delivery. J Neurooncol 89:159-168, 2008

50. Pardridge WM: Blood-brain barrier delivery. Drug Discov Today 12:54-61, 2007

51. Patel SJ, Shapiro WR, Laske DW, Jensen RL, Asher AL, Wessels BW, et al: Safety and feasibility of convectionenhanced delivery of Cotara for the treatment of malignant glioma: initial experience in 51 patients. Neurosurgery 56:1243-1253, 2005

52. Raghavan R, Brady ML, Rodríguez-Ponce MI, Hartlep A, Pedain C, Sampson JH: Convection-enhanced delivery of therapeutics for brain disease, and its optimization. Neurosurg Focus 20(4):E12, 2006

53. Rainov NG, Heidecke V: Long term survival in a patient with recurrent malignant glioma treated with intratumoral infusion of an IL4-targeted toxin (NBI-3001). J Neurooncol 66:197-201, 2004

54. Ram Z, Culver KW, Oshiro EM, Viola JJ, DeVroom HL, Otto E, et al: Therapy of malignant brain tumors by intratumoral implantation of retroviral vector-producing cells. Nat Med 3:1354-1361, 1997

55. Rand RW, Kreitman RJ, Patronas N, Varricchio F, Pastan I, Puri RK: Intratumoral administration of recombinant circularly permuted interleukin-4-Pseudomonas exotoxin in patients with high-grade glioma. Clin Cancer Res 6:21572165,2000

56. Ren H, Boulikas T, Lundstrom K, Söling A, Warnke PC, Rainov NG: Immunogene therapy of recurrent glioblastoma multiforme with a liposomally encapsulated replicationincompetent Semliki forest virus vector carrying the human interleukin-12 gene-a phase I/II clinical protocol. J Neurooncol 64:147-154, 2003

57. Richardson RM, Gimenez F, Salegio EA, Su X, Bringas J, Berger MS, et al: T2 imaging in monitoring of intraparenchymal real-time convection-enhanced delivery. Neurosurgery 69:154-163, 2011

58. Saito R, Bringas JR, Panner A, Tamas M, Pieper RO, Berger MS, et al: Convection-enhanced delivery of tumor necrosis factor-related apoptosis-inducing ligand with systemic administration of temozolomide prolongs survival in an intracranial glioblastoma xenograft model. Cancer Res 64:6858-6862, 2004

59. Saito R, Krauze MT, Noble CO, Tamas M, Drummond DC, Kirpotin DB, et al: Tissue affinity of the infusate affects the distribution volume during convection-enhanced delivery into rodent brains: implications for local drug delivery. J Neurosci Methods 154:225-232, 2006

60. Saito R, Sonoda Y, Kumabe T, Nagamatsu K, Watanabe M, Tominaga T: Regression of recurrent glioblastoma infiltrating the brainstem after convection-enhanced delivery of nimustine hydrochloride. J Neurosurg Pediatr 7:522-526, 2011

61. Salegio EA, Streeter H, Dube N, Hadaczek P, Samaranch L, Kells AP, et al: Distribution of nanoparticles throughout the cerebral cortex of rodents and non-human primates: Implications for gene and drug therapy. Front Neuroanat 8:9, 2014

62. Samaranch L, Salegio EA, San Sebastian W, Kells AP, Bringas JR, Forsayeth J, et al: Strong cortical and spinal cord transduction after AAV7 and AAV9 delivery into the cerebrospinal fluid of nonhuman primates. Hum Gene Ther 24:526-532, 2013

63. Sampson JH, Akabani G, Archer GE, Berger MS, Coleman $\mathrm{RE}$, Friedman AH, et al: Intracerebral infusion of an EGFRtargeted toxin in recurrent malignant brain tumors. Neuro Oncol 10:320-329, 2008

64. Sampson JH, Brady M, Raghavan R, Mehta AI, Friedman $\mathrm{AH}$, Reardon DA, et al: Colocalization of gadolinium-diethylene triamine pentaacetic acid with high-molecular-weight molecules after intracerebral convection-enhanced delivery in humans. Neurosurgery 69:668-676, 2011

65. Sampson JH, Raghavan R, Provenzale JM, Croteau D, Reardon DA, Coleman RE, et al: Induction of hyperintense signal on T2-weighted MR images correlates with infusion distribution from intracerebral convection-enhanced delivery of a tumor-targeted cytotoxin. AJR Am J Roentgenol 188:703709, 2007

66. Sampson JH, Reardon DA, Friedman AH, Friedman HS, Coleman RE, McLendon RE, et al: Sustained radiographic and clinical response in patient with bifrontal recurrent glioblastoma multiforme with intracerebral infusion of the recombinant targeted toxin TP-38: case study. Neuro Oncol 7:90-96, 2005

67. Sarka L, Burai L, Brücher E: The rates of the exchange reactions between [Gd(DTPA) $]^{2-}$ and the endogenous ions $\mathrm{Cu}^{2+}$ and $\mathrm{Zn}^{2+}$ : a kinetic model for the prediction of the in vivo stability of [Gd(DTPA) $]^{2-}$, used as a contrast agent in magnetic resonance imaging. Chemistry 6:719-724, 2000

68. Shahar T, Ram Z, Kanner AA: Convection-enhanced delivery catheter placements for high-grade gliomas: complications and pitfalls. J Neurooncol 107:373-378, 2012

69. Smith JH, Humphrey JA: Interstitial transport and transvascular fluid exchange during infusion into brain and tumor tissue. Microvasc Res 73:58-73, 2007

70. Sonabend AM, Stuart RM, Yun J, Yanagihara T, Mohajed $\mathrm{H}$, Dashnaw S, et al: Prolonged intracerebral convection-enhanced delivery of topotecan with a subcutaneously implantable infusion pump. Neuro Oncol 13:886-893, 2011

71. Stupp R, Mason WP, van den Bent MJ, Weller M, Fisher $\mathrm{B}$, Taphoorn MJ, et al: Radiotherapy plus concomitant and adjuvant temozolomide for glioblastoma. $\mathbf{N}$ Engl J Med 352:987-996, 2005

72. Syková E, Nicholson C: Diffusion in brain extracellular space. Physiol Rev 88:1277-1340, 2008

73. Szoka F Jr, Papahadjopoulos D: Comparative properties and methods of preparation of lipid vesicles (liposomes). Annu Rev Biophys Bioeng 9:467-508, 1980

74. Valles F, Fiandaca MS, Bringas J, Dickinson P, LeCouteur R, Higgins R, et al: Anatomic compression caused by highvolume convection-enhanced delivery to the brain. Neurosurgery 65:579-586, 2009

75. Varenika V, Dickinson P, Bringas J, LeCouteur R, Higgins R, Park J, et al: Detection of infusate leakage in the brain using real-time imaging of convection-enhanced delivery. J Neurosurg 109:874-880, 2008

76. Vargová L, Homola A, Zámecník J, Tichý M, Benes V, Syková E: Diffusion parameters of the extracellular space in human gliomas. Glia 42:77-88, 2003

77. Vogelbaum MA, Sampson JH, Kunwar S, Chang SM, Shaffrey M, Asher AL, et al: Convection-enhanced delivery of cintredekin besudotox (interleukin-13-PE38QQR) followed by radiation therapy with and without temozolomide in newly diagnosed malignant gliomas: phase 1 study of final safety results. Neurosurgery 61:1031-1038, 2007

78. Voges J, Reszka R, Gossmann A, Dittmar C, Richter R, Garlip G, et al: Imaging-guided convection-enhanced delivery and gene therapy of glioblastoma. Ann Neurol 54:479-487, 2003 
79. Wang H, Zhao P, Liang X, Gong X, Song T, Niu R, et al: Folate-PEG coated cationic modified chitosan-cholesterol liposomes for tumor-targeted drug delivery. Biomaterials 31:4129-4138, 2010

80. Wang W, Sivakumar W, Torres S, Jhaveri N, Vaikari VP, Gong A, et al: Effects of convection-enhanced delivery of bevacizumab on survival of glioma-bearing animals. Neurosurg Focus 38(3):E8, 2015

81. Weaver M, Laske DW: Transferrin receptor ligand-targeted toxin conjugate (Tf-CRM107) for therapy of malignant gliomas. J Neurooncol 65:3-13, 2003

82. Weber F, Asher A, Bucholz R, Berger M, Prados M, Chang S, et al: Safety, tolerability, and tumor response of IL4Pseudomonas exotoxin (NBI-3001) in patients with recurrent malignant glioma. J Neurooncol 64:125-137, 2003

83. White E, Bienemann A, Malone J, Megraw L, Bunnun C, Wyatt M, et al: An evaluation of the relationships between catheter design and tissue mechanics in achieving high-flow convection-enhanced delivery. J Neurosci Methods 199:8797, 2011

84. Yin D, Forsayeth J, Bankiewicz KS: Optimized cannula design and placement for convection-enhanced delivery in rat striatum. J Neurosci Methods 187:46-51, 2010

85. Zhu J, Zhang L, Hanisch UK, Felgner PL, Reszka R: A con- tinuous intracerebral gene delivery system for in vivo liposome-mediated gene therapy. Gene Ther 3:472-476, 1996

\section{Disclosures}

The authors report no conflict of interest concerning the materials or methods used in this study or the findings specified in this paper.

\section{Author Contributions}

Conception and design: Aghi, Jahangiri. Acquisition of data: Jahangiri, Chen. Analysis and interpretation of data: Jahangiri, Chin. Drafting the article: Jahangiri, Chin. Critically revising the article: Aghi, Flanigan, Chen, Bankiewicz. Reviewed submitted version of manuscript: Aghi, Jahangiri, Flanigan. Approved the final version of the manuscript on behalf of all authors: Aghi.

\section{Correspondence}

Manish K. Aghi, UCSF Neurological Surgery, 505 Parnassus Ave., Rm. M779, San Francisco, CA 94143-0112. email: manish. aghi@ucsf.edu. 